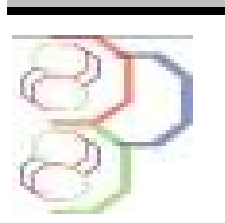

Journal of Applied Biosciences 97:9227 - 9239

ISSN 1997-5902

\title{
Analyse de la fiabilité des statistiques des pêcheries maritimes artisanales togolaises générées par la méthode ARTFISH de la FAO
}

\author{
SEDZRO Kossi Maxoe ${ }^{1}$ *, FIOGBE Emile Didier ${ }^{1}$, GUERRA Eduardo Balguerias ${ }^{2}$, STAMATOPOULOS \\ Constantine ${ }^{3}$ \\ ${ }^{1}$ Laboratoire de Recherche sur les Zones Humides, Faculté des Sciences et des Techniques, Université d'Abomey- \\ Calavi, 01BP526 Cotonou Bénin, Tél. (229) 95268262 \\ ${ }^{2}$ Institut Espagnol de l'Océanographie (IEO) - Espagne \\ ${ }^{3}$ Consultant International, Ancien Fonctionnaire de la FAO. \\ *Auteur correspondant au 03BP 30295 Lomé 03 -TOGO ; E-mail: ksedzro69@hotmail.com ;Tél: +228 90070333.
}

Original submitted in on $4^{\text {th }}$ November 2015. Published online at www.m.elewa.org on $31^{\text {st }}$ January 2016 http://dx.doi.org/10.4314/jab.v97i1.8

\section{RESUME :}

Objectif: L'objectif de la présente étude est d'évaluer la précision et l'exactitude des données sur les indicateurs des pêcheries artisanales maritimes togolaises en vue de leur utilisation en connaissance de cause. Méthodologie et résulta : La collecte et le traitement des données primaires sur les variables de ces pêcheries (effort de pêche, captures, CPUE, etc.) s'effectuent depuis 1999 suivant un programme d'enquêtes utilisant un logiciel. Les deux éléments sont connus sous le nom de "ARTFISH". Les données secondaires mensuelles de variabilité générées à l'aide d'ARTFISH (niveaux d'exactitude, coefficients de variation et coefficients d'activité pirogue) et stockées de 1999 à 2012 dans une base à la Direction des pêches du Togo (DPA) sont extraites à $38,55 \%$ par échantillonnage et évaluées à partir des tests statistiques et de l'analyse en composantes principales (ACP). Les résultats obtenus montrent que les estimations de l'effort de pêche et des captures sont respectivement exactes à $96,94 \%$ et $85,71 \%$ et précises à $95,92 \%$ et $57,14 \%$ et ; celles de la CPUE sont précises à $72,45 \%$. Les forts coefficients de variation qui se situent entre 16,65 et 36,90\% s'expliqueraient par l'hétérogénéité observée souvent dans les débarquements des captures. Le coefficient d'activité pirogue variant de 29,53 à $70,09 \%$ dépasse $100 \%$ de juillet à décembre surtout sur le site « Port de pêche» et les pêcheries de la senne tournante.

Conclusion et application des résultats : Il est retenu que les estimations de l'effort de pêche, des captures, de la CPUE, etc. des pêcheries artisanales maritimes du Togo sont fiables au moins à $57,14 \%$ des échantillons. Toutefois, les forts coefficients de variation estimés à $42,86 \%$ des échantillons et attribuables à l'hétérogénéité des débarquements des captures, montreraient la rareté des ressources halieutiques des eaux togolaises. Ainsi, les estimations des indicateurs des pêcheries artisanales maritimes du Togo peuvent-elles être utilisées sans trop de restrictions.

Mots clés : Togo, pêcheries artisanales maritimes, ARTFISH, estimations, données, précision, exactitude. 
Sedzro et al. J. Appl. Biosci. 2016 Analyse de la fiabilité des statistiques des pêcheries maritimes artisanales togolaises générées par la méthode ARTFISH de la FAO

Liability Analysis of Togolese marine artisanal fisheries statistics produced with ARTFISH FAO method

\begin{abstract}
Objective: The objective of this study is to assess the precision and accuracy of the Togolese maritime artisanal fisheries data indicators for their use knowingly.

Methodology and results: Data on fishing effort catch, CPUE (catch per unit effort), catches and values of catches of the Togolese marine artisanal fisheries was collected and processed following a program and software of FAO (Food and Agriculture Organization of the United Nations) called "Approaches, Rules and Techniques in fisheries (ARTFISH)" and stocked in the Fisheries Department Database. The secondary data, from 1999 to 2012 on the parameters of precision (correlation coefficient) and accuracy (accuracy levels) on the fishing effort, catches and canoes activity coefficient were extracted at $38.55 \%$ by sampling from this database and assessed with statistic tests and the principal component analysis (PCA). The results of the analysis showed that, the estimates of the accuracy of fishing effort and catches were accurate respectively at $96.94 \%$ and $85.71 \%$ of the samples. The variation in fishing effort, CPUE and catches were accurate to $95.92 \%$; $72.45 \%$ and $57.14 \%$ of samples respectively. The higher coefficients of variation ranging from 16.65 to $36.90 \%$ could be explained by the heterogeneity observed in the landings of catches. The canoe activity coefficients (CAC) ranging from 29.53 to $70.09 \%$ of the different gear exceed $100 \%$ from July to December, especially in the minor stratum "Fishing Harbor" and the purses seine (PS) fisheries.

Conclusion and application of results: It is retained that estimates of fishing effort, catches, CPUE etc. of Togo marine artisanal fisheries are reliable at least $57.14 \%$ of the samples. However, the higher coefficient of variation at $42.86 \%$ of the samples and due to the heterogeneity of catches landings would show the scarcity of the fishery resources in Togo waters. So the fisheries indicators estimated can be used without too many restrictions.
\end{abstract}

Keywords: Togo, marine artisanal fisheries, ARTFISH, estimations, data, precision, accuracy.

\section{INTRODUCTION}

Les pêcheries artisanales maritimes, définies par la FAO (1999) comme étant la somme de toutes les activités halieutiques portant sur une ressource donnée (pêcherie de merlus ou de crevettes) ou les activités d'un type ou mode d'exploitation unique d'une ressource particulière (pêcherie à la senne littorale), sont les plus actives des pêcheries au Togo depuis des décennies (FAO/UN, 1966 ; Horemans, 1996 ; Sedzro, 2006). Les travaux menés sur ces pêcheries artisanales ont, presque tous, posé le problème de manque d'informations suffisantes et fiables (Faggianelli et Faggianelli, 1984 ; Weigel et Hem, 1984; Samba, 1986 ; Bernacsek et al, 1987 ; Horemans, 1996 ; Denke, 1997 ; Sedzro, 2006 ; FAO, 2007 ; Sedzro, 2009 et 2014) pour la prise de toute décision de gestion durable des ressources halieutiques (Pauly, 2009). II en serait de même pour les pays voisins du Togo (FAO EAF-Nansen Project/FAO, 2014), peut-être pas avec la même acuité. La recherche de solution à ce problème a amené l'État Togolais, avec l'appui de la FAO dans le cadre du projet : TCP/RAF/0070 (T) (Sedzro, 2002), à mettre en place depuis 1999 un système de collecte et de traitement des données, basé sur un programme d'enquêtes et un logiciel. Ce système connu sous l'« Approche des règles et techniques dans la pêche (ARTFISH)» (Stamatopoulos, 2003 et 2009) produit des données sur les pêcheries artisanales maritimes. La présente étude a pour objectif d'évaluer, pour la première fois, la fiabilité de ces données en vue de leurs utilisations sereines pour des actions d'aménagement des pêcheries, entre autres. 


\section{Sedzro et al. J. Appl. Biosci. 2016 Analyse de la fiabilité des statistiques des pêcheries maritimes artisanales togolaises générées par la méthode ARTFISH de la FAO}

\section{MATERIEL ET METHODES}

Zone d'étude : Situé en Afrique de l'Ouest, le Togo dont les pays voisins sont le Ghana, le Bénin et le Burkina s'ouvre sur l'océan Atlantique. Son littoral s'étire le long du $6^{\circ} 5^{\prime}$ latitude nord et de $1^{\circ} 10^{\prime}$ à $1^{\circ} 40^{\prime}$ longitude est et sa côte de $50 \mathrm{~km}$ de long abrite une vingtaine de campements (PNAE/PSGL, 2001) où sont débarqués les produits des pêcheries artisanales maritimes qui s'opèrent sur un plateau continental étroit de $1500 \mathrm{~km}^{2}$ (carte1).

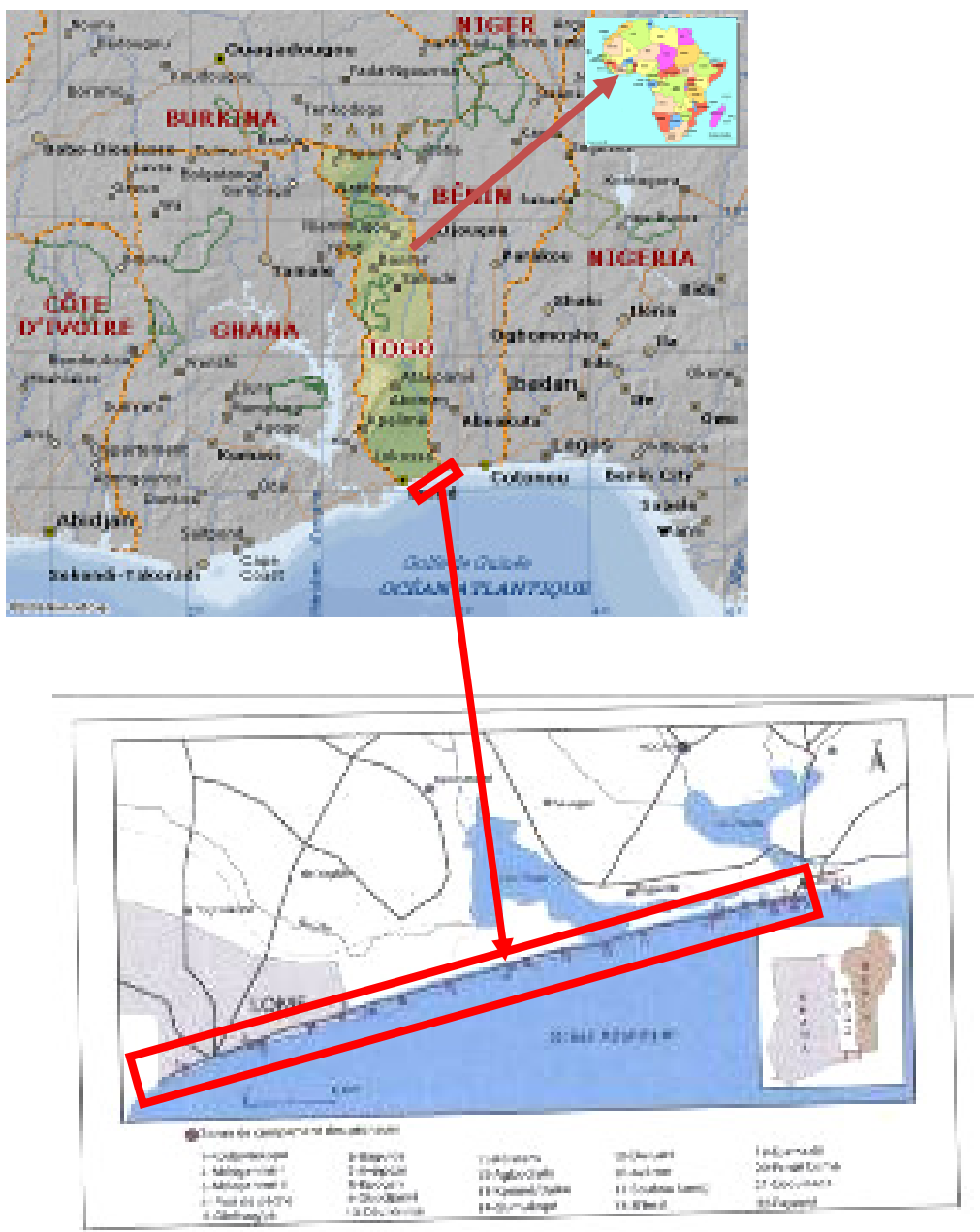

Carte 1 : Localisation de la zone d'étude

Collecte et saisies des données primaires dans

"ARTFISH" au Togo: Nous effectuons la collecte et la saisie des données d'effort de pêche, de captures et de valeur des prises par espèce ou groupe d'espèces et par type d'engin : senne tournante (ST), senne de plage (SP), filet maillant de surface (FMS), filet maillant de fond (FMF), filet flottant (FF), filet à requin (FR) et ligne, depuis 1999 à travers 4 catégories d'enquêtes conformément au programme ARTFISH (Sedzro, 2002). II s'agit des :

(i) Enquêtes-cadres s'effectuant une fois par an et portant sur le recensement exhaustif de la structure des pêches artisanales maritimes (pêcheurs, pirogues, engins, mareyeuses) et des espèces pêchées et classées à partir des fiches d'identification (Schneider, 1992 ; Murai et al., 2003). Elles permettent d'élaborer un plan d'échantillonnage avec le découpage de la côte en strates majeures et mineures comportant des sites d'échantillonnage et l'établissement de la variable pirogue-engins ;

(ii) Enquêtes sur les jours actifs, permettant de connaitre les jours où des activités de pêche ont eu lieu dans les sites d'échantillonnage. Elle contribue à l'estimation de l'effort de pêche et autres ; 

artisanales togolaises générées par la méthode ARTFISH de la FAO

(iii) Enquêtes sur le nombre de pirogues-engins actives pendant les jours de pêche dans les sites d'échantillonnage. Elle contribue à l'estimation de l'effort de pêche exprimé en jours-pirogue ;

(iv) Enquêtes sur les débarquements des espèces ou groupe d'espèces par des pirogues-engins par un échantillonnage aléatoire simple dans les siteséchantillons pour déterminer les CPUE globales.

Le logiciel "ARTFISH" a 2 composantes ("ArtBasic" et "ArtSer"). L'ArtBasic sert à l'enregistrement des données d'enquêtes-cadres et d'échantillonnage et à l'estimation mensuelle des captures, d'effort de pêche, de CPUE, des prix moyens et des valeurs des captures, des niveaux d'exactitude, des variabilités desdites variables et des coefficients d'activité pirogue (CAP) par strate mineure et l'ArtSer à l'agrégation par an desdites variables.

Estimation des données de capture, de l'effort et de la CPUE : La formule générique utilisée pour l'estimation de la capture totale dans une strate mineure dans un mois suivant l'approche d'échantillonnage dans l'espace et dans le temps (Stamatopoulos, 2003 et 2009) est: Capture $=$ CPUE $\times$ Effort où CPUE est la capture par unité d'effort de pêche Effortobtenue dans la strate mineure pendant le mois.

\section{Capture $=$ CPUE $\times$ Effort}

\section{où}

CPUE est la capture par unité d'effort de pêche Effort obtenue dans la strate mineure pendant le mois.

L'estimation de la CPUE globale d'une strate mineure dans un mois donné : Les captures débarquées sont réparties en tas par espèce. Le nombre, le poids et le prix des tas sont enregistrés avant la première vente par les enquêteurs et la CPUE globale est estimée suivant la formule :

$$
\text { CPUE }=\frac{1}{m} \sum_{j=1}^{m} \sum_{i=1}^{n} Q i j
$$

où

Qij est la quantité des prises de l'espèce ou groupe d'espèces $\mathbf{i}$ de la pirogue-engin-jour $\mathbf{j}$ échantillonnée ; $\mathbf{n}$ est le nombre total d'espèces ou groupe d'espèces débarquées par la pirogue-engin-jour j échantillonnée ; m est le nombre total de pirogue-engin-jour $\mathbf{j}$ échantillonnées dans une strate mineure pendant le mois. L'estimation de l'effort de pêche total dans une strate mineure et dans un mois donné : Elle est donnée par la formule (Stamatopoulos, 2009): Effort $=$ CAP $\times$ F $\times$ A

Où

Elle est donnée par la formule (Stamatopoulos, 2009): Effort $=$ CAP $\times$ F $\times$ A où CAP est le coefficient d'activité pirogue-engin ou probabilité qu'une pirogue-engin de pêche soit active pendant un jour donné ; $\mathbf{F}$ obtenu par des enquêtes-cadres est un facteur d'extrapolation, exprimant le nombre total de pirogues-engin actives dans une strate mineure; $\mathbf{A}$ un facteur d'extrapolation temporelle exprimant le nombre de jours d'activités de pêche dans une strate mineure pendant le mois.

L'estimation du coefficient d'activité pirogue-engin (CAP)

La formule ci-contre est appliquée pour estimer le CAP :

$$
\mathbf{C A P}=\frac{\sum_{i=1}^{\mathbf{Y}} \mathbf{P i}}{\mathbf{Y} \times \sum_{i=1}^{\mathrm{k}} \mathbf{R i}} \quad \text { où }
$$

Pi le nombre de pirogues-engins i actives dans tous les $\mathbf{k}$ sites d'échantillonnage pendant l'un des $\mathbf{Y}$ jours d'échantillonnage dans une strate mineure; $\mathbf{R i}$ le nombre total de pirogues-engins i recensées par une enquête-cadre dans l'un des $\mathbf{k}$ sites d'échantillonnage d'une strate mineure ; $\mathbf{Y}$ le nombre total de jours d'échantillonnage dans tous les $\mathbf{k}$ sites d'échantillonnage. A partir de toutes ces formules, la capture totale dans la strate mineure s'exprime par

$$
: \text { Capture }=\frac{1}{\mathrm{~m}} \sum_{\mathrm{j}=1}^{\mathrm{m}} \sum_{\mathrm{i}=1}^{\mathrm{n}} Q \mathrm{ij} \times \frac{\sum_{\mathrm{i}=1}^{\mathrm{P}} \mathrm{Pi}}{\mathrm{Yx}_{\mathrm{x}=1}^{\mathrm{k}} \mathrm{Ri}} \times \mathrm{F} \times \mathrm{A}
$$

La capture totale ou production annuelle dans toutes les strates majeures est calculée grâce à 


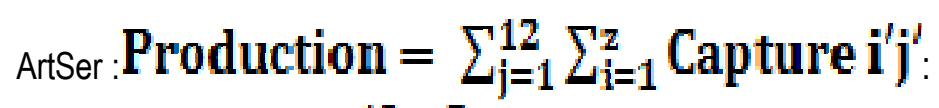

Production $=\sum_{j=1}^{12} \sum_{i=1}^{2}$ Capturei'j'

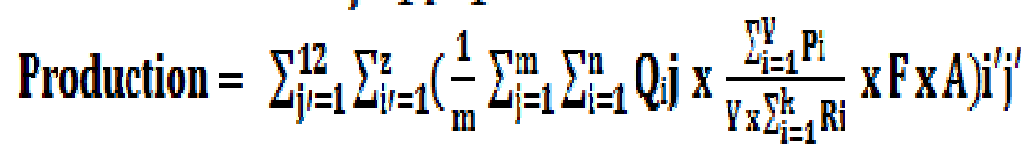

où $\mathbf{z}$ est le nombre total des strates mineures.

La précision de l'échantillonnage est mesurée par le coefficient de variation $\left(\mathbf{C}_{\mathrm{v}}\right)(\%)$ de formule :

$$
\mathrm{C}_{\mathrm{v}}=\frac{\boldsymbol{\sigma}}{\mathbf{m}}
$$

Où $\boldsymbol{\sigma}$ estl'écart type des variables mesurées et $\mathbf{m}$ est le moyen échantillonnage.

L'estimation du niveau d'exactitude ( $\mathrm{Ne}$ ) : L'exactitude de l'échantillonnage (Ne ou A) exprimée par un indice relatif en pourcentage (0 à 100\%), indique le degré de proximité entre le paramètre estimé par échantillonnage et celui de la population vraie. La formule (Stamatopoulos, 2003 et BRAND GMBH, 2014) est :

$$
N_{e}=1-\frac{|\mu-m|}{\operatorname{Max}-\operatorname{Min}}
$$

où

$$
\mathrm{N}_{\mathrm{e}}(\%)=\frac{\mathbf{m}-\mu}{\mu} 100
$$

Max-Min est l'amplitude de la population; $\mathbf{m}$ est la moyenne échantillonnale et $\mu$ est la moyenne nominale de la population vraie. Transformée en format normalisé, elle devient :

$$
\mathbf{N}_{0}=1-|\mathbf{m}-\mu| \text {. }
$$

Analyse des données de capture, de l'effort de pêche et de la CPUE : Le tableau 1 présente les mois des années de 1999 à 2012 dont les données sont échantillonnées et analysées pour l'évaluation de la fiabilité des données de l'effort de pêche, de la CPUE, des captures et des valeurs des captures. Ces données sont les moyennes mensuelles et annuelles par type d'engin et par strate mineure des niveaux d'exactitude Ne des captures (NEC) et d'effort de pêche (NEEP), des coefficients de variation de l'effort de pêche (VEP),

\begin{tabular}{|c|c|c|c|c|c|c|c|c|c|c|c|c|c|c|}
\hline $\begin{array}{l}\text { Années } \\
\text { Mois tête de } \\
\text { série }\end{array}$ & 1999 & 2000 & 2001 & 2002 & 2003 & 2004 & 2005 & 2006 & 2007 & 2008 & 2009 & 2010 & 2011 & 2012 \\
\hline Janvier & Janvier & Février & Mars & Avril & Mai & Juin & Juillet & Août & Septembre & Octobre & Novembre & Décembre & Janvier & Février \\
\hline Avril & Avril & Mai & Juin & Juillet & Août & Septembre & Octobre & \begin{tabular}{|l|} 
Novembre \\
\end{tabular} & Décembre & Janvier & Février & Mars & Avril & Mai \\
\hline \begin{tabular}{|l|} 
Juillet \\
\end{tabular} & Juillet & Août & Septembre & Octobre & Novembre & Décembre & Janvier & Février & Mars & Avril & Mai & Juin & Juillet & Août \\
\hline Octobre & Octobre & Novembre & Décembre & Janvier & Février & Mars & Avril & Mai & Juin & Juillet & Août & Septembre & Octobre & \begin{tabular}{|l|} 
Novembre \\
\end{tabular} \\
\hline Décembre & Décembre & Janvier & Février & Mars & Avril & Mai & Juin & Juillet & Août & Septembre & Octobre & Novembre & Décembre & Janvier \\
\hline Mars & Mars & Avril & Mai & Juin & Juillet & Août & Septembre & Octobre & Novembre & Décembre & Janvier & Février & Mars & Avril \\
\hline
\end{tabular}
de la CPUE (VCPUE) et des captures (VC) et des coefficients d'activité pirogue (CAP).

Tableau 1 : Répartition des mois échantillonnés suivant les différentes années

Les tests de corrélation, de corrélation sous méthode de Spearman, de Kolmogorov Smirnov, de Bartlett, de Student, de Wilcoxon et de Linaer model sont utilisés. L'analyse en composantes principales (ACP) et les logiciels R version 2.14.0 (De MICHEAUX et al., 2010) et Excel sont également utilisés. 


\section{RESULTATS}

Les 22 sites de débarquement des produits de la pêche sont regroupés dans trois states mineures: strates mineures Port, strate mineure Hors Port et strate mineure Lacs qui sont réparties dans deux strates majeures: strates majeures Golfe et Lacs. La taille des échantillons des données de paramètres de précision, d'exactitude et de CAP est de 4608 soit $38,55 \%$ de la population de 11952 couvrant 84 des 168 mois de 1999 à 2012 et tous les segments de pêche ou engins de pêche : la ST, la $\mathrm{SP}$, le FMS, le FMF, le FF, le FR et la ligne. Le test de Kolmogorov-Smirnov ( $p$-value $>0,05$ ) montre que les échantillons de chacune des variables (NEEP, NEC, VEP, VCPUE, VC et CAP) suivent une distribution normale et le test de Student et de Fisher ( $p$-value $<0,05)$ montrent de différence significative entre elles, à l'exception du couple VCPUE et VC ( $p$-value $=0,085$ ). Analyse des paramètres d'exactitude: La figure 1 présente les niveaux d'exactitude moyens annuels des échantillons par segment (NEEP et NEC). Pour le NEEP, 96,94\% des échantillons ont des niveaux d'exactitude variant de 90 à $99,8 \%$ et $3,06 \%$ des échantillons de $87,29 \%$ à $89,72 \%$. Pour le NEC, $85,71 \%$ des échantillons ont des scores compris entre 90,38\% et $98,83 \%$ alors que $14,29 \%$ ont des niveaux d'exactitude de $82,88 \%$ à $89,91 \%$.
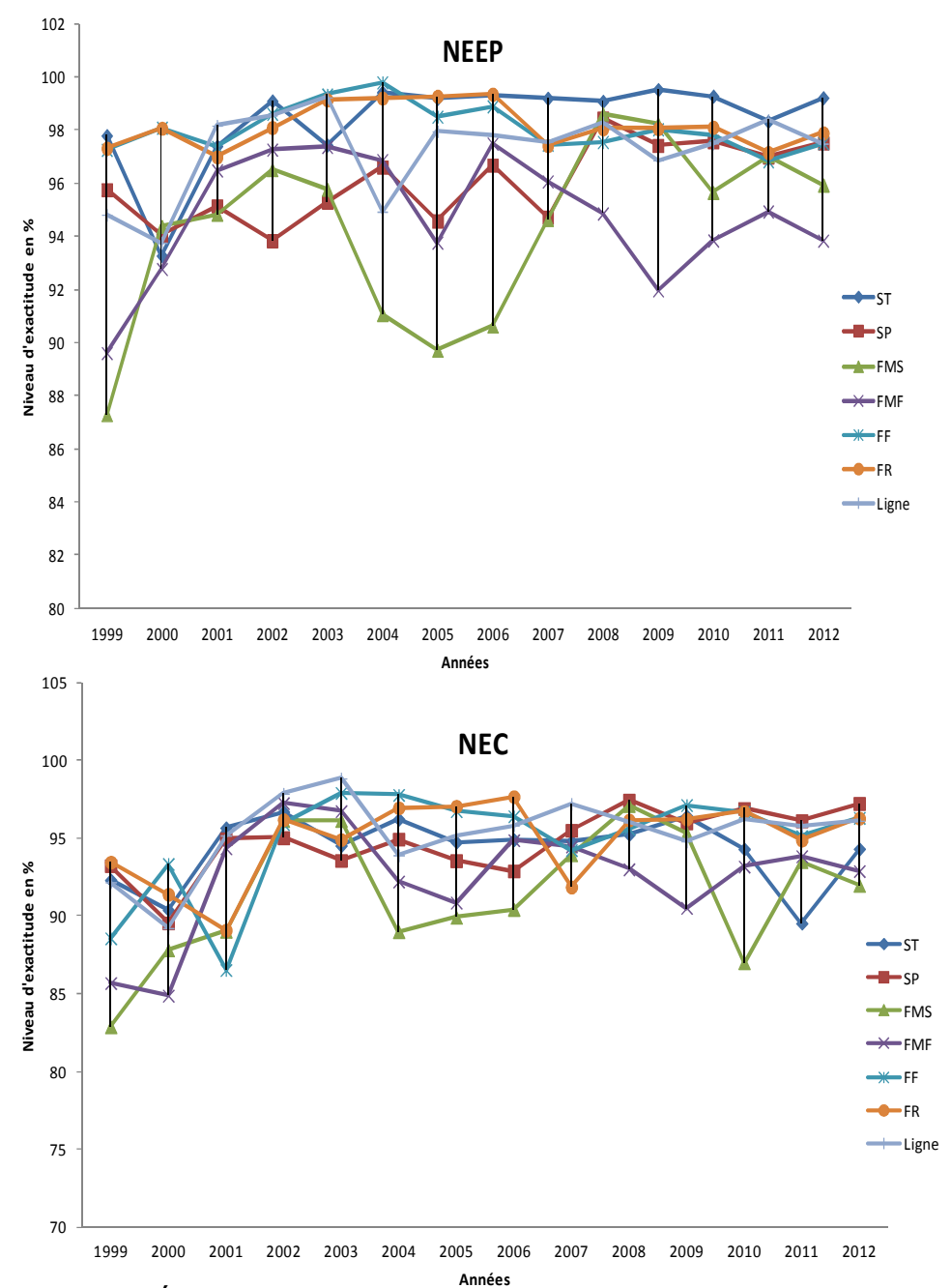

Figure 1 : Évolution des moyennes annuelles des niveaux d'exactitude de l'effort et des captures par engin de pêche de 1999 à 2012 des pêcheries artisanales maritimes togolaises

Analyse des paramètres de précision: La figure 2 montre que 53,06 à $95,92 \%$ des échantillons ont des coefficients de variation moyens annuels inférieurs ou égaux à $16 \%$; tandis que (i) $4,08 \%$ des échantillons ont 
des scores variant de $16 \%$ et $20,58 \%$ (VEP) ; (ii) 29,59\% ont des scores se situant entre 16 et $33 \%$ et $1,02 \%$ ont un score de $33,91 \%$ (VCPUE) et (iii) $45,92 \%$ avec des scores de 16 à $33 \%$ et 1,02 ont un score de $36,90 \%$ (VC).
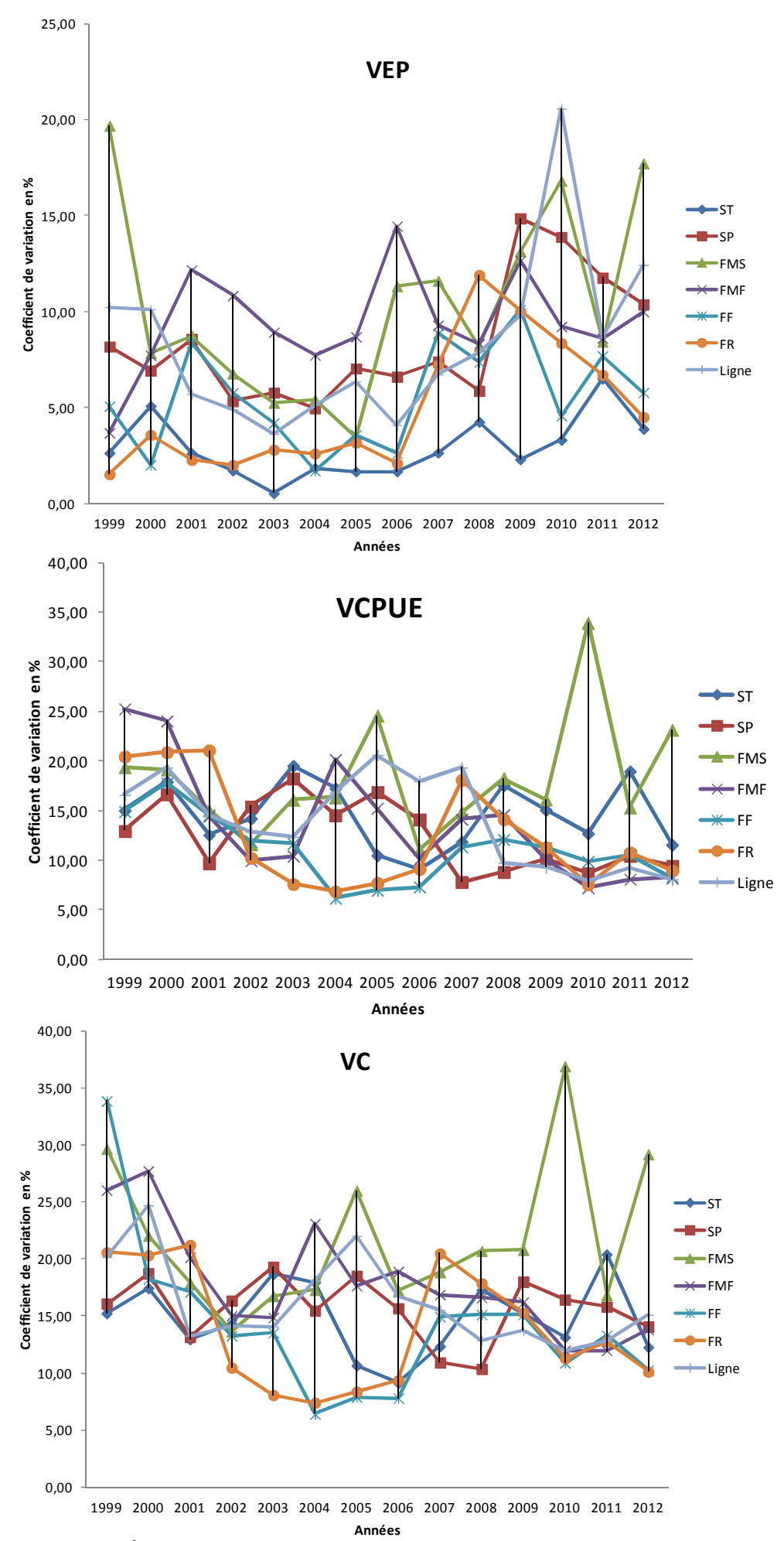

Figure 2 : Évolution des moyennes annuelles des variabilités de l'effort, de la CPUE et des captures par engin de pêche de 1999 à 2012 des pêcheries artisanales maritimes togolaises. 
Analyse du coefficient d'activité pirogue (CAP) : La figure 3 présente l'évolution des coefficients de d'activité pirogue moyens annuels. Une tendance à la baisse générale est observée à partir de 2007. Tous les scores se situent entre 97,5\% en 1999 (FR) et 10,4\% en 2008
(FMS) à l'exception du FMS dont le score est de 110,5\% en 2006. Globalement, la ST a enregistré les plus grands scores allant de $95,7 \%$ en 2003 à $52,4 \%$ en 2007 et le FF a eu les plus faibles compris entre $47 \%$ et 12,1 .

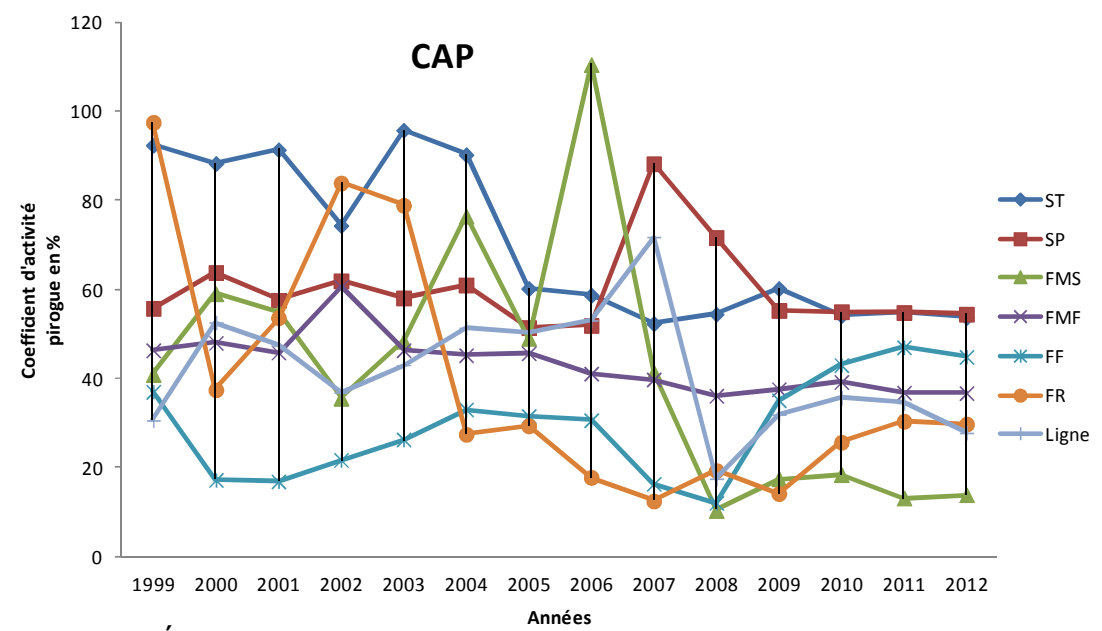

Figure 3 : Évolution des moyennes annuelles des coefficients d'activité pirogue par engin de pêche de 1999 à 2012 des pêcheries artisanales maritimes togolaises

Analyse des moyennes globales et des intervalles de confiance: Le tableau 2 et la figure 4 présentent les moyennes globales et les intervalles de confiances des différentes variables. Les moyennes globales des NEEP et des NEC sont toutes supérieures à $90 \%$ et les plus élevées sont respectivement de $98,40 \%$ avec $\mathrm{IC}_{0,95}$ de $\pm 0,86$ (ST) et de 95,30 avec $I C_{0,95}$ de $\pm 1,25$ (Ligne). Les moyennes globales des VEP, VCPUE et VC varient respectivement de $2,92 \pm 0,83$ à $10,32 \% \pm 2,60 ; 11,05 \pm$ 1,71 à $18,20 \% \pm 3,08$ et $13,83 \pm 2,76$ à $21,69 \% \pm 3,39$ et le CAP de $29,53 \pm 5,97$ à 70,09\% $\pm 9,18$.

Tableau 2 : Moyennes globales et intervalle de confiance du niveau d'exactitude et de variabilité de l'effort de pêche, des captures et de la CPUE et du coefficient d'activité pirogue en pourcentage

\begin{tabular}{|c|c|c|c|c|c|c|c|c|c|c|c|c|}
\hline & \multicolumn{2}{|c|}{ Niv d'exact effort } & \multicolumn{2}{|c|}{ Niv d'exact capture } & \multicolumn{2}{|c|}{ Variabilité effort } & \multicolumn{2}{|c|}{ Variabilité capture } & \multicolumn{2}{|c|}{ CAP } & \multicolumn{2}{|c|}{ Variabilité CPUE } \\
\hline & Moyenne & $\mid C_{0,95}$ & Moyenne & $I C_{0,95}$ & Moyenne & $I_{0,95}$ & Moyenne & $I C_{0,95}$ & Moyenne & $I_{0,95}$ & Moyenne & $I_{0,95}$ \\
\hline ST & 98,40 & $\pm 0,86$ & 94,29 & $\pm 1,12$ & 2,92 & $\pm 0,83$ & 14,79 & $\pm 1,71$ & 70,09 & $\pm 9,18$ & 14,55 & $\pm 1,74$ \\
\hline SP & 96,04 & $\pm 0,77$ & 94,78 & $\pm 1,10$ & 8,40 & $\pm 1,65$ & 15,64 & $\pm 1,43$ & 60,09 & $\pm 5,07$ & 12,42 & $\pm 1,85$ \\
\hline FMS & 94,31 & $\pm 1,76$ & 91,43 & $\pm 2,17$ & 10,32 & $\pm 2,60$ & 21,69 & $\pm 3,39$ & 42,13 & $\pm 14,70$ & 18,20 & $\pm 3,08$ \\
\hline FMF & 94,79 & $\pm 1,22$ & 92,48 & $\pm 1,88$ & 9,46 & $\pm 1,35$ & 17,92 & $\pm 2,54$ & 43,27 & $\pm 3,43$ & 13,72 & $\pm 3,04$ \\
\hline FF & 98,07 & $\pm 0,45$ & 94,87 & $\pm 1,77$ & 5,56 & $\pm 1,38$ & 14,12 & $\pm 3,51$ & 29,53 & $\pm 5,97$ & 11,05 & $\pm 1,71$ \\
\hline FR & 98,15 & $\pm 0,42$ & 94,92 & $\pm 1,33$ & 4,92 & $\pm 1,75$ & 13,83 & $\pm 2,76$ & 39,89 & $\pm 14,48$ & 12,49 & $\pm 2,82$ \\
\hline Ligne & 97,23 & $\pm 0,84$ & 95,30 & $\pm 1,25$ & 8,31 & $\pm 2,30$ & 16,09 & $\pm 2,01$ & 41,78 & $\pm 7,20$ & 13,91 & $\pm 2,3$ \\
\hline
\end{tabular}



artisanales togolaises générées par la méthode ARTFISH de la FAO

Les résultats du calcul des coefficients de corrélation entre les variables sont présentés dans le tableau 3. Les fortes corrélations $(0,87$ à $-0,91)$ sont observées entre VC et VCPUE ; NEEP et NEC ; NEEP et VEP etc. Les corrélations moyennes $(0,79$ à $-0,58)$ sont obtenues entre VEP et VC; NEC et VCPUE; etc. Les faibles corrélations $(0,44$ à $-0,31)$ se retrouvent entre VEP et VCPUE ; CAP et VCPUE et autres. Les résultats du test de corrélation ( $p$-value $<0,05)$ montrent que les liens existant entre les couples de variables NEEP-VEP, NEEP-NEC, etc. ne seraient pas accidentels (tableau 3).

Tableau 3 : Corrélation entre les variables des 7 pêcheries artisanales togolaises

\begin{tabular}{|l|l|l|l|l|r|r|r|}
\hline Variables & CAP & & NEEP & VEP & NEC & VC & \multicolumn{1}{|l|}{ VCPUE } \\
\hline CAP & & 1,00 & 0,09 & $-0,31$ & 0,05 & $-0,08$ & 0,19 \\
\hline NEEP & & 1,00 & $-0,91$ & 0,83 & $-0,89$ & $-0,60$ \\
\hline VEP & & & & 1,00 & $-0,58$ & 0,79 & 0,44 \\
\hline NEC & & & & & 1,00 & $-0,90$ & $-0,77$ \\
\hline VC & & & & & & 1,00 & 0,87 \\
\hline VCPUE & & & & & & & 1,00 \\
\hline
\end{tabular}

Les résultats du déterminant $(-2,19 \mathrm{e}-05)$, du test de Bartlett $(\mathrm{df}=5, \mathrm{p}$-value $=2.135 \mathrm{e}-09$ ) et de l'indice de $\mathrm{KMO}=0,6$ ont permis d'initier l'ACP. Au total, 6 composantes principales sont déterminées mais, deux expliquant $87,8 \%$ de la variance totale (CP1 $=67,7 \%$ et $\mathrm{CP} 2=20,1 \%$ ) sont retenues (tableau 4 et Fig. 4). Le premier axe factoriel CP1, "Axe d'exactitude et de précision" porte les variables qui lui sont corrélées et qui comprennent NEEP $(21,96 \%)$ et NEC $(20,38)$ opposées à VC $(24,10 \%)$; VEP $(17,20 \%)$ et à VCPUE $(16,10 \%)$. Elles contribuent ensemble à $99,74 \%$ à la formation dudit axe. Le deuxième axe factoriel CP2 "Axe d'activités ou de pression" porte la variable CAP qui lui est fortement corrélée et contribue elle seule à $71,34 \%$ à la formation de cet axe.

Tableau 4 : Contribution des variables à la formation des axes factoriels

\begin{tabular}{|l|r|r|}
\hline & Comp 1 & Comp 2 \\
\hline CAP & 0,27 & 71,34 \\
\hline NEEP & 21,96 & 0,86 \\
\hline VEP & 17,20 & 11,51 \\
\hline NEC & 20,38 & 1,19 \\
\hline VC & 24,10 & 0,25 \\
\hline VCPUE & 16,10 & 14,86 \\
\hline
\end{tabular}

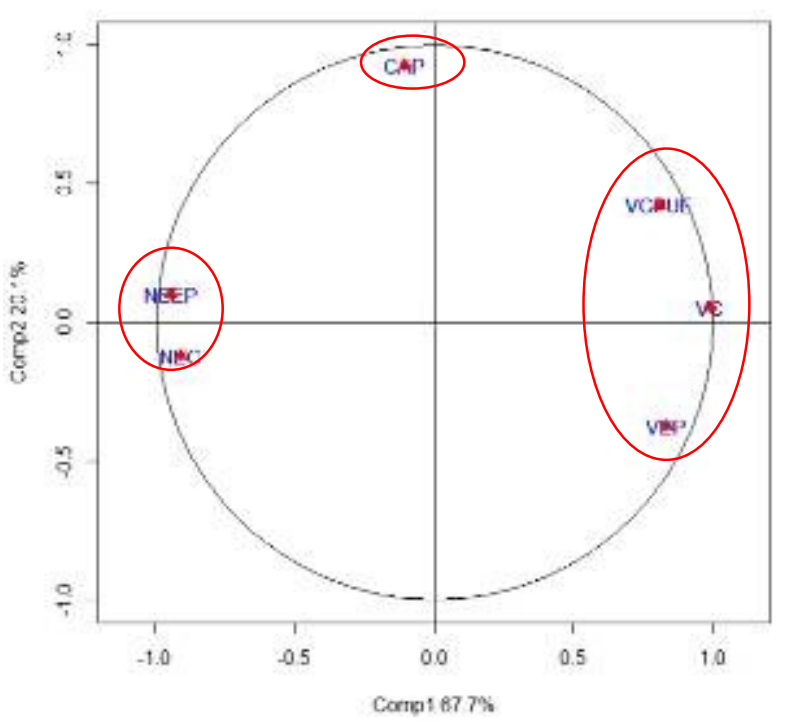

Figure 4 : Représentation des variables dans Comp1xComp2

Le tableau 5 et la figure 5 présentent les 7 composantes des pêcheries : la ST, la SP, le FMF, le FMS, le FR, le FF et la ligne. Ils montrent que la composante FMS contribuant à la CP1 (56,67 \%) explique à elle seule plus de la $1 / 2$ de la variance de cet axe. Les composantes ST et $\mathrm{FF}$ contribuant respectivement à $63,97 \%$ et à $25,16 \%$ 

artisanales togolaises générées par la méthode ARTFISH de la FAO

à la $\mathrm{CP} 2$, expliquent à elles seules plus de $8 / 9$ de la variance du deuxième axe.

Tableau 5 : Contribution des individus à la formation des axes factoriels

\begin{tabular}{|l|r|r|}
\hline & \multicolumn{1}{|l|}{ Comp 1 } & \multicolumn{1}{c|}{ Comp 2 } \\
\hline ST & 8,31 & 63,97 \\
\hline SP & 0,28 & 1,54 \\
\hline FMS & 56,67 & 0,70 \\
\hline FMF & 11,31 & 3,11 \\
\hline FF & 11,65 & 25,16 \\
\hline FR & 11,29 & 2,10 \\
\hline Ligne & 0,51 & 3,42 \\
\hline
\end{tabular}

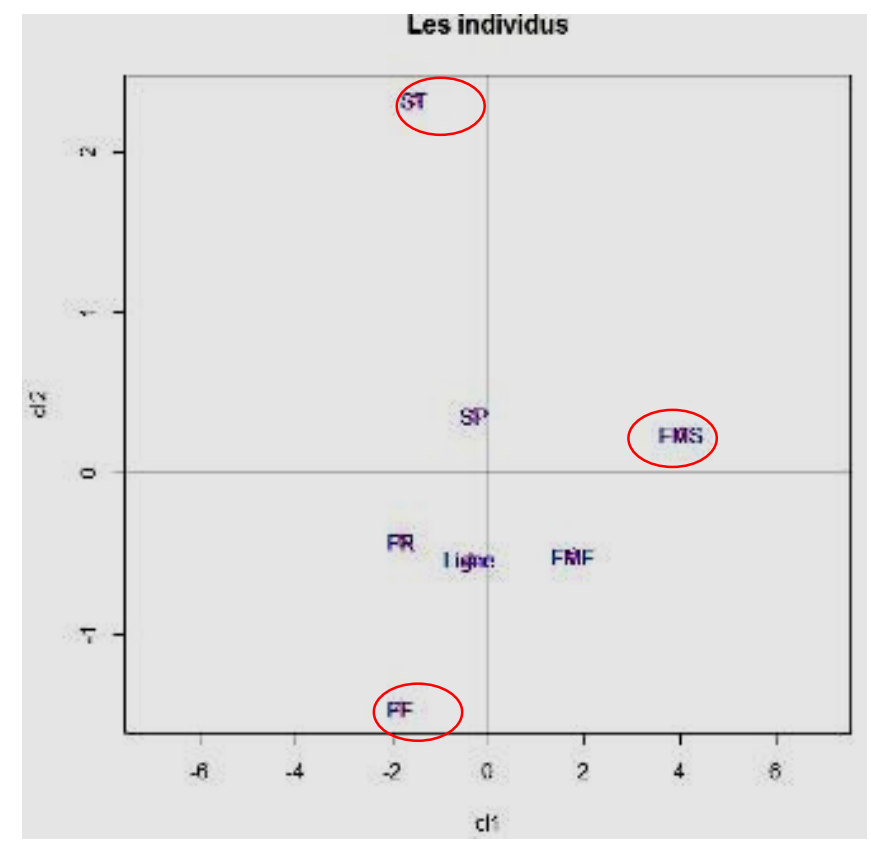

Figure 5 : Représentation graphique des individus dans $\mathrm{CP} 1 \times \mathrm{CP} 2$

\section{DISCUSSIONS}

Les résultats obtenus (Fig. 1) montrent que les estimations des variables sur l'effort de pêche, la CPUE, les captures, etc. peuvent être considérées exactes conformément aux exigences de ARTFISH selon lesquelles, le niveau d'exactitude minimal requis pour que l'estimé de la moyenne des échantillons soit proche de celui de la population vraie est de $90 \%$ (Stamatopoulos, 2003 et 2009). En effet pour le NEEP, 96,94\% des échantillons ont des niveaux d'exactitude variant de 90 à
$99,8 \%$ et pour le NEC, $85,71 \%$ des échantillons ont des scores entre 90,38 et $98,83 \%$. Le moyen échantillonnage global estimé sur les 14 ans pour l'effort de pêche et des captures (toutes catégories d'engins et de strates mineures confondues) est plus proche de celle de la population vraie (tableau 2 et 4 et Fig. 4). Les niveaux d'exactitude inférieurs à $90 \%$ observés portent majoritairement sur les estimations des variables dans la strate mineure "Lacs » où le nombre d'échantillons 


\section{Sedzro et al. J. Appl. Biosci. 2016 Analyse de la fiabilité des statistiques des pêcheries maritimes}

artisanales togolaises générées par la méthode ARTFISH de la FAO

requis n'est souvent pas atteint surtout au début de l'introduction d'ARTFISH en 1999 au Togo.

En référence à l'interprétation du coefficient de variation (cv) de Martin et al., (2004), Baillargeon (2008) et Statistique Canada (2009), les résultats montrent (Fig. 2) que les estimations de :

L'effort de pêche (VEP) seraient précises à 95,92\% des échantillons (cv compris entre 0,55 et $14,86 \%$ ) et comporteraient d'erreurs d'échantillonnage à $4,02 \%$ des échantillons (cv compris entre 16,81 et $20,58 \%)$;

La CPUE (VCPUE), seraient précises à $72,45 \%$ des échantillons de cV compris entre 6,18 et $16,58 \% ; 26,53 \%$ comporteraient d'erreurs avec cv variant de 16,65 à $24,56 \%$. Elles ne seraient pas précises à 1,02\% des échantillons, le cv étant égal à 33,91\% ;

- $\quad$ La capture (VC), seraient précises à $57,14 \%$ des échantillons (cv de 6,41 à 16,62\%) ; comporteraient d'erreurs à $40,82 \%$ des échantillons dont le cv est de 16,70 à $29,66 \%$ et ne seraient pas du tout précises à $2,04 \%$ des échantillons de cv égal à $33,84 \%$ et à $36,90 \%$. Les estimations des variables dont le cv se situent entre 16,70 et $29,66 \%$ peuvent faire l'objet d'utilisation prudente (Martin et al., 2004; Baillargeon, 2008 ; Statistique Canada, 2009) ; mais, Stamatopoulos (2009) estime que seules les estimations dont le cv est inférieurs ou égal à $15 \%$ présentent des signes d'homogénéité dans les mesures et sont donc acceptables. Ainsi, pourrions-nous conclure que les estimations concernant l'effort de pêche seraient toujours précises à 95,92\% des échantillons ; mais, celles de la CPUE à $65,31 \%$, et les captures à 41,84\%. Cependant, les cv allant de 15 ou 16,65 à 36,90\% qui semblent exprimer des estimations erronées, s'expliqueraient par l'effet d'hétérogénéité (Baillargeon, 2008) observée dans les débarquements de tous les engins. II est observé donc qu'en 1999 le FMS a débarqué en moyenne 369,5 $\mathrm{kg}$ par marée avec un maximum de $882 \mathrm{~kg}$ et un minimum de $22 \mathrm{~kg}$ et en 2010, 414,25 kg avec un pic de $4575 \mathrm{~kg}$ et un minimum de $18 \mathrm{~kg}$ et le FF a débarqué en moyenne 591,64 kg par marée avec un maximum de $1974 \mathrm{~kg}$ et un minimum de $20 \mathrm{~kg}$. Quant à la Ligne, le débarquement moyen en 2000 est de 43,66 kg avec un maximum de $870 \mathrm{~kg}$ et un minimum de $2 \mathrm{~kg}$. Les résultats

\section{CONCLUSION}

Les estimations de l'effort de pêche sont exactes à $96,94 \%$ des échantillons et des captures à $85,71 \%$. Celles de l'effort de pêche, de la CPUE et des captures sont précises respectivement à $95,92 \% ; 72,45 \%$ et $57,14 \%$ des échantillons cependant, les forts coefficients montrent également que le CAP varie de 29,53 à 70,09\% suivant les catégories d'engin de pêche (tableau 2) et a dépassé en 2006 les 100\% pour le FMS (Fig. 3). Pour les pirogues de la ST, le CAP varie entre $102,5 \%$ et $167 \%$ de juillet à novembre-décembre dans la strate mineure "Port». Les causes sont l'arrivée pendant la "haute" saison de pêche dans les pêcheries togolaises d'un grand nombre des pirogues des pêcheurs migrants ghanéens (Atti-Mama, 1991 ; DIPA, 1991 ; Denke, 1997 ; Tvedten et al., 1997; Sedzro, 2009) après le recensement des facteurs de production effectué souvent en début d'année. Le CAP, globalement bas pour certaines catégories de pêcheries (Fig. 3) avec la faible taille de leur flottille (25 pirogues de FF, 29 de Ligne et 39 de FMS), expliquerait les écarts observés plus haut au niveau des cv allant de 15 ou 16,65 à 36,90\%. L'analyse de la fiabilité des données a, permis de déterminer des caractéristiques des pêcheries artisanales maritimes togolaises. Aussi, la pêcherie du FF serait-elle la moins active (CAP de $29,53 \% \pm 5,97 \%$ ) et celle de la ST la plus active (CAP de $70,09 \% \pm 9,18 \%$ ) avec un plus grand effort de pêche moyen annuel de 22533 jours-pirogues. Cette dernière par le CAP exercerait plus de pression sur les ressources halieutiques des eaux togolaises et interpelle les autorités chargées des pêches dans leur rôle régalien (Guilland, 1979). Les résultats du test de corrélation et de la fonction "Linear model", (tableau 3) montrent qu'il n'existerait de relation réelle de "cause à effet" qu'entre trois couples de variables seulement :

$$
\begin{aligned}
& \text { NEEP }=0,999 * \mathrm{NEC}+2,9033 \\
& \mathrm{VEP}=0,78 * \mathrm{VC}-5,5 \\
& \mathrm{VC}=1,05 * \mathrm{VCPUE}+1,89 .
\end{aligned}
$$

Ces résultats corroborent avec ceux de Stamatopoulos (2003 et 2009) et de Baillargeon (2008) selon lesquels la précision et l'exactitude sont deux paramètres distincts l'un de l'autre; mais les plus utilisées en science pour déterminer l'erreur liée à une mesure (Socrates Mine RVA Programme, 2002) et pour apprécier la qualité des données (Gouvernement du Canada, 2002 ; DrzevieckiRenard et al., 2006 ; Afristat, 2009).

de variation de 16,65 à 36,90 s'expliqueraient par l'hétérogénéité observée dans les débarquements des captures. Cette dispersion est observée au niveau des quantités débarquées dont les extrêmes sont de 0,10 $\mathrm{kg}$ et de $5000 \mathrm{~kg}$ suivant les segments de pêche et 


\section{Sedzro et al. J. Appl. Biosci. 2016 Analyse de la fiabilité des statistiques des pêcheries maritimes}

artisanales togolaises générées par la méthode ARTFISH de la FAO

montrerait la rareté des ressources. Le CAP global de 29,53 à $70,09 \%$ et atteignant parfois $160 \%$ de juillet à décembre surtout dans la strate mineure "Port» s'expliquerait par le phénomène de forte migration des pêcheurs surtout de la senne tournante. Les liens réels existant entre les couples NEEP-NEC, VEP-VC et VC-

\section{REMERCIEMENTS}

Les auteurs remercient la Direction des pêches et de l'Aquaculture et les enquêteurs pour les efforts fournis en

\section{REFERENCES BIBLIOGRAPHIQUES}

Afristat, 2009. Problématique de la qualité des données statistiques. Revue du Schéma directeur de la Statistique du Mali. Présentation PPT. INSTAT du Mali, Bamako. 30 diapos.

Atti-Mama C, 1991. Migrations de pêcheurs au Bénin et au Togo, in Migrations des pêcheurs en Afrique de l'Ouest. Programme DIPA, Cotonou. DIPA/WP/36.pp 259-273.

Baillargeon J, 2008. Application et interprétation des techniques statistiques avancées. Répertoire FTP /pub/dpsy/baillarg/srp6018. Décanat des études de cycles supérieurs et de recherche. Université du Québec. $6 p$.

Bernacsek GM, Faggianelli DJ, Amegavie GK et Lhomme $F, 1987$. Profil des ressources halieutiques du Togo. FAO, COPACE/TECH/87/82, 91 p.

BRAND GMBH + CO KG, 2014. Gestion de qualité Exactitude. Que signifient dans la mesure volumétrique limite d'erreur, exactitude, coefficient de variation et précision ? 3p.

Denke AM, 1997. Enquête-cadre et socioéconomique sur la pêche artisanale maritime au Togo. Programme pour le Développement Intégré des Pêches Artisanales en Afrique de l'Ouest, Cotonou, Bénin, DIPA/WP/102, 71p.

DIPA, 1991. Migration des pêcheurs en Afrique de l'Ouest, DIPA/WP/36, 332pp

Drzeviecki-Renard S et Gensse M, 2006. La qualité en biochimie - précision et exactitude. $7 p$. http://pedagogie.acmartinique.fr/stv/bioch/Qualit\%C3\%A9.pdf

Faggianelli DJ et Faggianelli E, 1984. La pêche maritime artisanale au Togo : caractéristiques générales et résultats statistiques pour la saison 1983-84. Doc. Centre ORSTOM Lomé, 83p.

FAO, 1999.Directives techniques pour une pêche responsable. No. 4. Rome Italie, FAO. 91p. http://www.fao.org/docrep/003/w4230f/w4230f08 .htm\#TopOfPage 21 décembre 2012FAO,
VCPUE montrent bien l'indépendance entre les paramètres de précision et d'exactitude. Enfin, par ces résultats nous pouvons conclure que les estimations des indicateurs sur les pêcheries artisanales maritimes du Togo pourraient être utilisées sans trop de restrictions.

vue de la collecte et du traitement des données sur les pêcheries artisanales maritimes du Togo.

2007. Profil de pêche par pays, République Togolaise. (FAO FID/CP/TGO). N ${ }^{\circ} X X X$. Rome. FAO, 2007.34 p.

FAO, 2010. Appui à l'amélioration de la gestion de la pêche à la senne de plage au Bénin, Côte d'Ivoire, Ghana et Togo. Projet (EAF-Nansen GCP/INT/003/NOR).http://www.eafnansen.org/nansen/topic/18211/fr.

FAO EAF-Nansen Project/FAO, Projet EAF-Nansen, 2014. Establishing a baseline for the implementation of an ecosystem approach to marine fisheries management in West and Central Africa/Etablissement des éléments de référence pour la mise en œuvre d'une approche écosystémique de la gestion des pêches maritimes en Afrique de l'Ouest et centrale. FAO EAF-Nansen Project Report/FAO Rapport du projet EAF-Nansen. No.20. Rome, FAO. 2014. 89 p.

FAO/UNDP, 1966. Rapport au Gouvernement du Togo sur le développement et l'organisation de l'industrie des pêches. Basé sur le travail de W.H.L. Allsopp. Rep. FAO/UNDP(TA), (TA 2184) : Italie, 19p. http://www.fao.org/docrep/005/45187f/45187F02 .htm\#res5.

Gouvernement du Canada, 2002. Cadre de gestion de la qualité des données de l'inventaire national des rejets de polluants. 37p. http://www.ec.gc.ca/inrpnpri/default.asp?lang=Fr\&n=23EAF55A-1 le 29 août 2014 à $19 \mathrm{~h}$

Horemans B, 1996. Revue Sectorielle, Politique de Développement et Programme d'action, 1996. Définition d'une politique et d'un plan d'action pour la pêche. Projet TCP/TOG/34. 54p.

http://education.ti.com/sites/FRANCE/downloads/pdf/dec ouverte ti83p.pdf.http://www.brand.de/fr/informa tions-techniques/gestion-de-qualite/exactitude/ 


\section{Sedzro et al. J. Appl. Biosci. 2016 Analyse de la fiabilité des statistiques des pêcheries maritimes}

artisanales togolaises générées par la méthode ARTFISH de la FAO

Lafaye De Micheaux P, Drouilhet R, and Liquet B, 2010. Le logiciel R, Maitriser le langage, Effectuer des analyses statistiques Springer, 2010 (Software : $R$ Package, le Logiciel $R$, version 1.2) http://cran.r-

roject.org/web/packages/LeLogicielR/citation.ht $\underline{\mathrm{ml}}$

Muraï T, d'Almeida AFM et Sohou Z, 2003. Atlas des Poissons et Crustacés du Bénin : Eaux marines. Préparé et publié à la Direction des Pêches avec la collaboration de la JICA. Cotonou, 188p.

Pauly D, 2009. Surpêche http://fr.wikipedia.org/wiki/Surp\%C3\%AAche consulté 15/08/12.

Pêches et Océans Canada, 2013. Enquête de 1990 sur la pêche récréative au Canada. Statistiques.7p.

PNAE-PSGL, 2001. Étude de faisabilité du projet de développement, d'outils de planification et de suivi de la gestion du littoral (PSGL), Rapport final, Agence Ecauch, $222 \mathrm{p}$.

Samba A, 1986. Collecte et traitement des statistiques de pêche artisanale au Gabon, au Cameroun et au Togo. Dakar, Programme COPACE, COPACE/TECH/86/77, $68 \mathrm{p}$.

Schneider W, 1992. Fiches FAO d'identification des espèces pour les besoins de la pêche. Guide de terrain des ressources marines commerciales $\mathrm{du}$ golfe de Guinée. Rome, Italie, FAO. 1992. 268p.

Sedzro KM, 2002. Appui à la mise en place de systèmes statistiques informatisés pour la pêche maritime au Togo. TCP/RAF - FAO 0070 (T). 51p.
Sedzro K M, 2009. Étude sur la migration des communautés de pêche dans la région du Comité des Pêches du Centre Ouest de Golfe de Guinée $54 \mathrm{p}$.

Sedzro KM, 2014. Rapport de référence pour l'aménagement de la pêcherie de la senne de plage au Togo. EAF-Nansen/FAO. Lomé, Togo, $57 p$

Socrates Mine RVA Programme, 2002. Une introduction aux statistiques sur TI-83. Exactitude et précision. $15 p$.

Stamatopoulos C, 2003.Safety in sampling. Methodological notes. FAO Fisheries Technical Paper. No. XXX. Rome, Italy. FAO, 2003. 92 p.

Stamatopoulos C, 2009. Prospections halieutiques par échantillonnage : manuel technique. FAO Document technique sur les pêches. No. 425. Rome, FAO. 2009. 142 p.

Statistique Canada, 2009. Exactitude et qualité des données. Enquête sur la sécurité financière de 2005 : guide de l'utilisateur pour le fichier de micros données à grande diffusion. $10 \mathrm{p}$.

Tvedten I and Hersoug B (Eds.) Fishing for Amadiume I, 1997. Re-Inventing Africa. Matriarchy, religion and culture. London: and Diaw, M. C. (Eds.) Fishermen's Migrations in West Africa. IDAF/WP/36.

Weigel JY et Hem S, 1984. Le secteur des pêches. Situation actuelle et perspective. République du Togo-Mission Française de Coopération LoméORSTOM Lomé, Togo, 85p. 